\title{
Dual Mechanism Inhibitor Control and Pharmacological Utility of Drug Resistance FLT3-ITD in Acute Myeloid Leukemia Cells
}

\author{
Maria Rogdaki ${ }^{1}$, Xinhua Xiao \\ ${ }^{* 1}$ Corresponding author: Department of Child and Adolescent Psychiatry, Institute of Psychiatry, Psychology and \\ Neuroscience, King's College, London, UK. E-mail: maria.rogdaki@kcl.ac.uk
}

Citation: Maria Rogdaki, Scatena A Dual Inhibitor Overcomes Drug-resistant FLT3-ITD Acute Myeloid Leukemia published in Med Science Journals.

Received Date: 02 August 2021 Accepted in Revised Form: 16 August 2021 Published Date: 04 September 2021

\begin{abstract}
FLT3 mutations are among the most common genetic alterations in acute-myeloid leukemia (AML). They are associated with poor prognosis. Multiple FLT3 inhibitors have been in clinical evaluation at various stages. Resistance to FLT3 inhibitors due to acq uired point mutations in the tyrosine-kinase domain (TKD), have limited the effectiveness of treatments. A "gatekeeper" mutation (F691L), is also resistant to most FLT3 inhibitors. New therapies are therefore needed.
\end{abstract}

FLT3 inhibitors are needed to protect against FLT3-TKD mutations and FLT3 internal tandem duplicate (FLT3-ITD). We identified KX2391, a dual FLT3/tubulin inhibitor, and examined its efficacy and mechanisms for overcoming drug-resistant FLT3ITD-TKD mutations. KX2391 had potent growth inhibitory effects and apoptosis promoting effects on AML cell lines that harbor FLT3-ITD mutations. KX2-391 orally administered significantly prolonged the survival time of a murine model with leukemia caused by FLT3ITD-F691L. KX2-391 also inhibited growth of primary AML cells that express FLT3ITD-F691L and 2 primary cells that are FLT3ITD-D835Y. Preclinical data suggest that KX2391 is a promising FLT3 inhibitor.

The treatment of AML patients with FLT3 mutations, particularly refractory/relapsed patients suffering from F691L or other FLT3TKD mutations.

Keywords: Acute Myeloid Leukemia, FLT3ITD, FLT3 Resistance Mutation, KX2-391, AC220

\section{Introduction}

FLT3 mutations are found in more than $30 \%$ patients with acute myeloidleukemia (AML). They are associated with short relapse-free survival and overall survival. This includes internal tandem duplications (ITD) as well as point mutations in the tyrosine kinasedomain (TKD). Multiple FLT3 kinase inhibitors have been identified and approved for clinical use. These include sorafenib and Gilteritinib. These drugs have a high rate of drug resistance and relapse, which can lead to transient clinical responses.

This can lead to disease progression and poor overall longevity. A number of mutations have been acquired in the TKD, and a "gatekeeper mutation" (F691L), is resistant to all FLT3 inhibitors currently available. F691L and other mutations can lead to drug resist ance. Therefore, it is critical that effective compounds are found. We identified KX2-391 FLT3 inhibitor, and tested its activity against FLT3ITD-TKD mutations using both in vitro and in-vivo models. To computationally screen 1487 small molecules ligands from L3400 Clinical Compound Library, we used molecular docking simulations. Previous studies had shown that KX2-391 was a potential FLT3 inhibitor. Our modeling predicted that KX2-391 would interact with FLT3's V624 and L616, which was also predicted by our modeling.

E661 residues. By cellular thermal shift analysis, we further confirmed the interactions between FLT3 \& KX2-391. Comparing with DMSO, there was a clear thermal shift in the melting curve. KX2-391 treatment resulted in detection of the protein. KX2-391 increased the thermal stability of FLT3 proteins in a dose-dependent way. KX2-391 had a potent inhibitory effect against FLT3 in Ba/F3 cell. It also inhibited growth of FLT3ITD-expressing Ba/F3 and all cells that were expressing FLT3ITD-TKD mutations. These cells are previously known to be drug resistant to FLT3 inhibitors like AC220. Ba/F3ITD-F691L cells showed a tenfold higher sensitivity to KX2$391(0.032 \mathrm{mM}$ vs. 0.372mM). KX2-391 had a higher inhibitory efficacy against human leukemia cells that harbor FLT3-ITD (MV4-11, MOLM13) than it did on FLT3-mutated leukemia cells. We observed a dose-dependent induction apoptosis of Ba/F3 cells that expressed FLT3-ITD, FLT3-ITD-D835Y, and FLT3-ITD-F691L. Also, we observed it in two FLT3-ITD positive AML cell line (Fig. 1e, f KX2391 prominently inhibited the phosphorylation of FLT3 and downstream targets STAT5, ERK and AKT in FLT3-ITD, FLT3-ITD-F691Lexpresssing Ba/F3 cells and other cells of our assay panel. KX2-391 is a tubulin/SRC inhibitor. We monitored SRC phosphorylation to assess KX2-391's effects on microtubule structure.

KX2-391 treatment didn't alter the phosphorylation SRC in FLT3 mutant cell lines (Fig. 1g, h). In MOLM13 cells, we detected disruptions 
in tubulin polymerization when treated with KX2-391 or with other tubulin inhibitors. Assays did not show that Vincristine, a tubulin inhibitor, had any effect on FLT3 phosphorylation or its downstream targets. To determine the efficacy of KX2-391, we used previously described Ba/F3/ITDF691L leukemia models to assess in vivo. KX2-391 had a significant effect on median survival.

It extended the survival time from 12 days to 23.5 days in comparison with the control group (Fig. 2a, P0.0001. Treatment did not cause significant weight loss or other toxicities. KX2-391-treated mice had less leukemia cells in peripheral blood, spleen and bone marrow than the AC220 or Gilteritinib group. They also had smaller spleens. H\&E staining showed that KX2-391 treatment significantly reduced the number of leukemia cells in peripheral blood and spleen.

AML cell infiltration into liver and spleen. KX2-391 also had anti-leukemia effects on primary AML cells taken from six newly diagnosed FLT3ITD AML patients. KX2-391 reduced cell viability in four primary blasts of FLT3-ITD mutations patients (comparable to AC220's effect It also significantly inhibited growth of two primary AML cells that expressed FLT3-ITD-D835Y - both of whom were resistant to AC220. KX2-391 didn't affect the growth of healthy peripheral mononuclear blood cells at the Similar concentrations. KX2-391 inhibited FLT3-phosphorylation in primary cells of AML. We conclude that KX2-391 is a strong FLT3/tubulin inhibitor which exerts strong therapeutic effects both in vitro \& in vivo against FLT3 -ITD and drug-resistant TKD mutations, including FLT3 -ITD-F691L. This mutation is the most difficult to overcome.

Clinically. KX2-391 could be a valuable second-line treatment for some of the most challenging AML cases due to its therapeutic effects and apparent low toxicity.

\section{Abbreviations}

AML: Acute Myelogenous Leukemia; BMCs; Bone marrow Cells; FLT3; FMS-like Tyrosine Kinase 3; ITD; Internal tandem duplication; TTKD: Tyrosinekinase domain; ESRK: Extracellular Signal regulated Kinase; STAT; Signal transducer \& activator of transcription; VCR; Vincristine; HMC: Half maximum inhibitory concentration; IC50; Sodium dodecyl and sin and eosin and eosin and sulfate;

\section{Data and materials availability}

All data generated and analyzed during the study are included in this article and its supplementary information.

\section{Declarations}

Participation requires ethical approval and consent. All animal experiments were approved and monitored by the Shanghai Jiao tong University School of Medicine Animal Care \& Welfare Committee. The Institutional Review Board of Shanghai Jiao tong University School of Medicine approved the preparation of patient samples.

\section{Competing interests}

The authors declare that they have no competing interests.

\section{Consent to publication}

It is not applicable. 


\section{References}

1. Lemonnier F, Inoue S, Mak TW. Acute myeloid genome classification, leukemia N Engl J Med. 2016;375(9).900-900.

2. Ley TJ. Miller C. Ding L. Raphael BJ. MungallAJ. Robertson AG. Hoadley K. Triche TJ Jr., Laird PW. Baty JD. et al. The genomic and epigenomic landscapes for adult de novo acutemyeloid leukemia. N Engl J Med. 2013;368(22):2059-74.

3. Asthana S, Olshen A, Travers KJ, et al. Single-cell analysis revealed heterogeneous resistance to quizartinib for acute myeloidleukemia. Blood. 2017;130(1), 48-58.

4. Eguchi M, Minami Y, Kuzume A, Chi S. Mechanisms underlying resistance, FLT3 inhibitors for acute myeloidleukemia Biomedicines. 2020;8(8).245.

5. Niu L, Yang J, Yan W, Yu Y, Zheng Y, Ye H, Chen Q, Chen L. Reversible. KXO1's low clinical toxicity is due to its binding to the colchicinebinding protein of b-tubulin. J Biol Chem. .2019;294(48):18099-108.

8. Anbalagan M, Carrier L, Glodowski S, Hangauer D, Shan B, Rowan BG. KX-01 is a novel inhibitor of Src kinase directed at the peptide substrate site, works in conjunction with tamoxifen for estrogen receptor alpha positive breasts, Cancer. Breast Cancer Res Treat. 2012;132(2), 391-409. 